\title{
INSPECTION OF INTERNAL STRUCTURE OF WALLS BY SUBSURFACE RADAR
}

\author{
A. Boryssenko, O. Boryssenko, A. Lishchenko, V. Prokhorenko \\ Research Co. "Diascarb" \\ P.O. Box 148, Kyiv, 02222, Ukraine \\ diascarb@public.ua.net
}

\begin{abstract}
This paper deals with the application of ultra-wide band time-domain subsurface radars, equipped with special signal processing techniques, to realize non-invasive image testing of the building walls' internal structure that are made with brick, stone, concrete, reinforced concrete and other construction materials. There are two normally associated problems, qualitative and quantitative, considered. Limitations and shortcomings of radar imaging, due to inherent physical features as well as signal processing improving the quality of radar images, are discussed. Actual field data are used to illustrate applications of subsurface radar for non-destructive testing of walls' internal regions.
\end{abstract}

Key words: Subsurface Radar, Ground Penetrating Radar, Nondestructive Testing, Radar Imaging

\section{INTRODUCTION}

Subsurface radar or ground penetrating radar (GPR) is worldwide effective non-invasive technique to study and image internal regions of opaque matters such as soils, rocks, brick, concrete, wood, fresh water, snow, ice, vegetation etc. Common GPR system includes three principle components: active sensor (transmitter and receiver with their antennas) spaced on surface that is border between air and media under investigation, and control electronics, and computer (Figure 1a).

The radar surveillance of testing areas is implemented by successive placing of the antenna sensor along a straight transect line in continues or stepped mode to implement the synthetic-aperture technique. The last enables to obtain preferable spatial resolution in radar where the used UWB antennas, especially in time-domain GPR, have inherently pure directive features (Finkelstein, 1994).

Return signals (A-scan) in each point, where the radar sensor is placed, are used to form a 2-D image of electromagnetic transect or the B-scan images. Various forms of image presentation due to computer's graphic opportunities can be used to visualize radar return like demonstrated in Figures $1 \mathrm{~b}$ and 1c. Moreover, practical radar data is strongly corrupted due to numerous unavoidable reasons. Therefore, computer processing and image enhancement must be implemented.
Subject of this study is an application of time-domain GPR of the 1-3 GHz band or equivalent $0.5-\mathrm{ns}$ sounding pulse to image internal structure of building's walls, columns, ceilings and others elements for their nondestructive testing (NDT). The authors have the corresponding practice in some projects in Ukraine (Boryssenko, 1999).

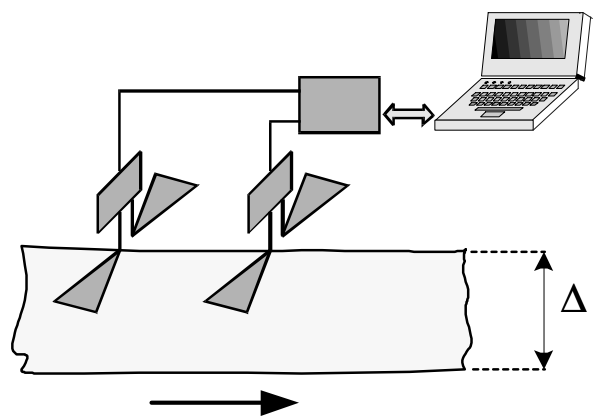

a)

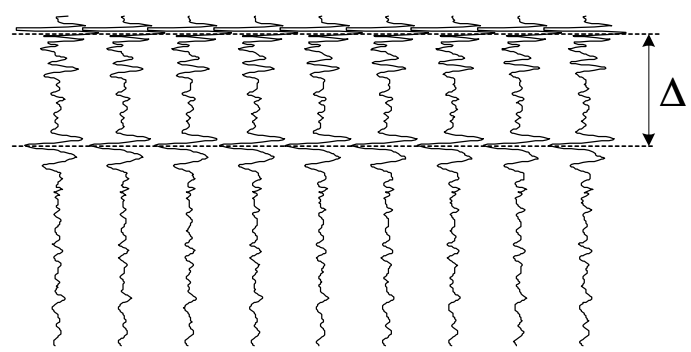

b)

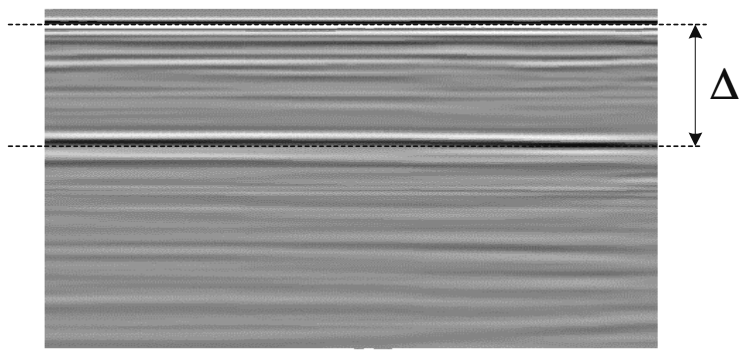

c)

Figure 1. (a) Basic principle of subsurface radar operation for wall structure imaging with the synthetic-aperture technique. (b-c) Visualizations of radar image for wall of $\Delta$ thickness. 


\section{PROBLEM DESCRIPTION}

Considered here problems should be solved for estimation physical and geometrical properties of walls, ceiling etc. by subsurface radar NDT. There are two possible levels of solutions, qualitative and quantitative. A qualitative level is based on visual analysis by expert estimation of the following features: presence and number of layers, availability of cracks and voids, presence of internal armature and reinforcement, stratification, hidden wires and tubes, and so on. Measurements of geometrical features, levels of humidity and corrosion of reinforcement etc. constitute the quantitative NDT studies.

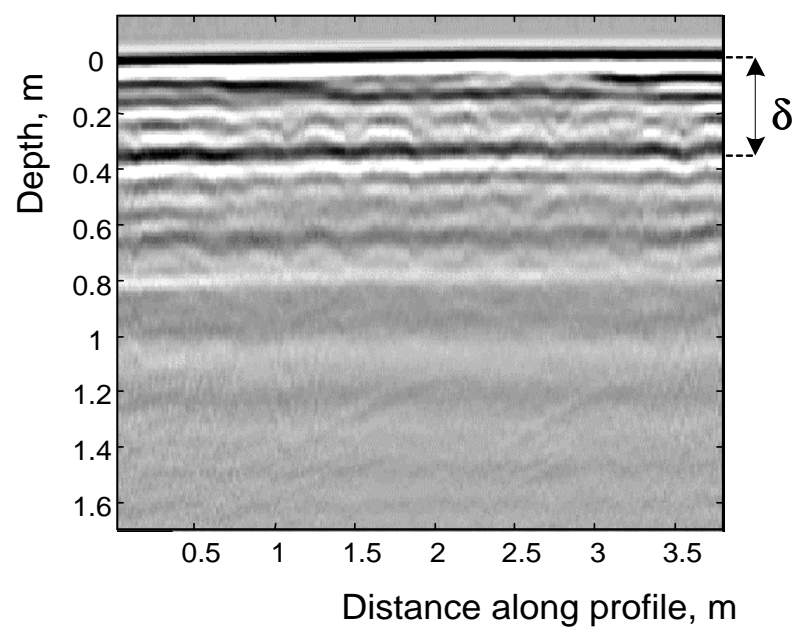

a)

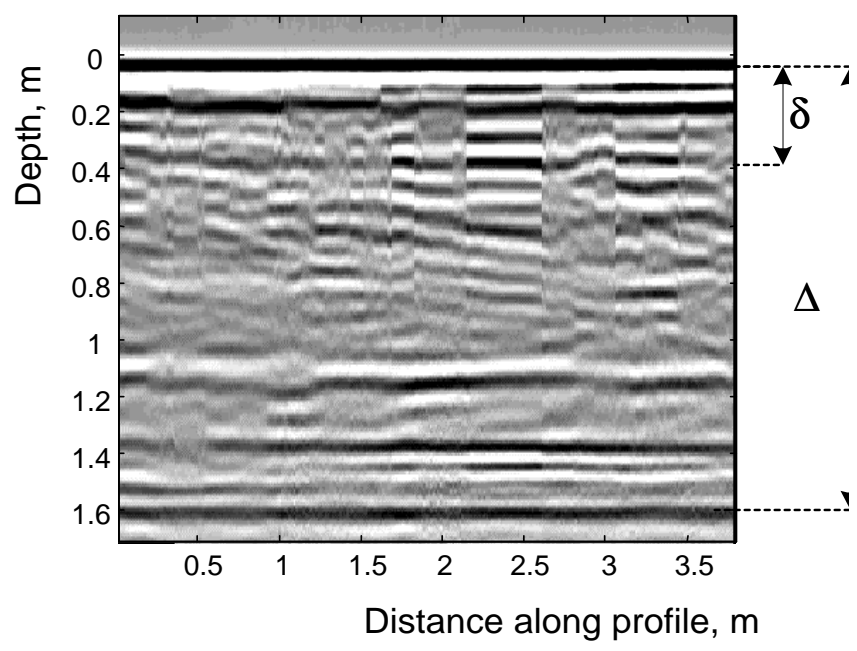

b)

Figure 2. GPR imaging of two columns with (a) strong signal attenuation and (b) low signal attenuation due to different internal humidity. The Central Portal of The Kyiv's National Olympic Stadium, Kyiv, Ukraine, 1998.
The example of qualitative NDT is in Figure 2 for two columns similar in their outward appearances. However Figure 2a demonstrates strong attenuation of sounding signal in the first column where the thickness $\delta$ of the first brickwork layer is only seen. The backside of the column diameter $\Delta$ is only fixed for the second column in Figure $2 b$. Such difference is forced by various internal humidity factors. Another kind of qualitative investigation is presented in Figure 3 to detect and locate by GPR an old marble column hidden inside a wall after set of reconstruction of the 16-century building. Similar problem to locate an old niche in building wall hidden under layer of plaster is depicted in Figure 4.

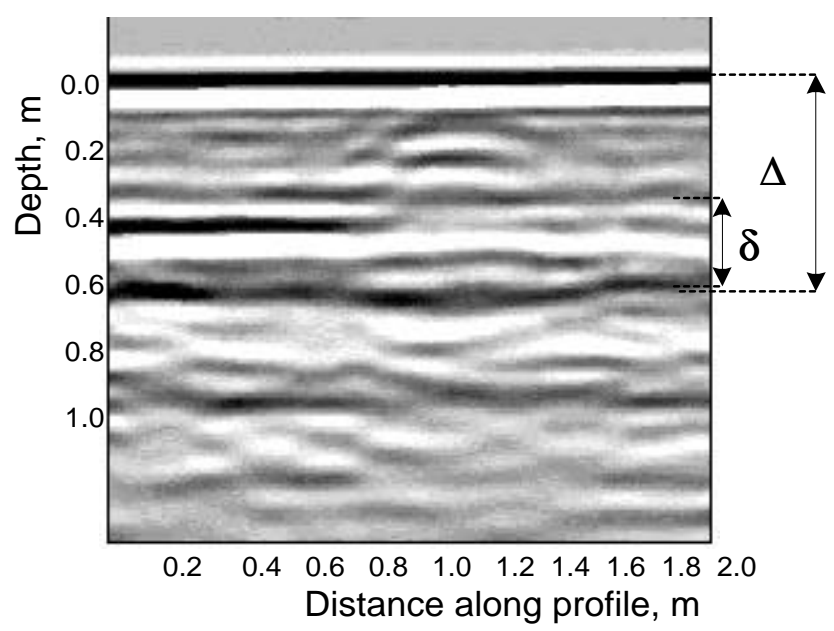

a)

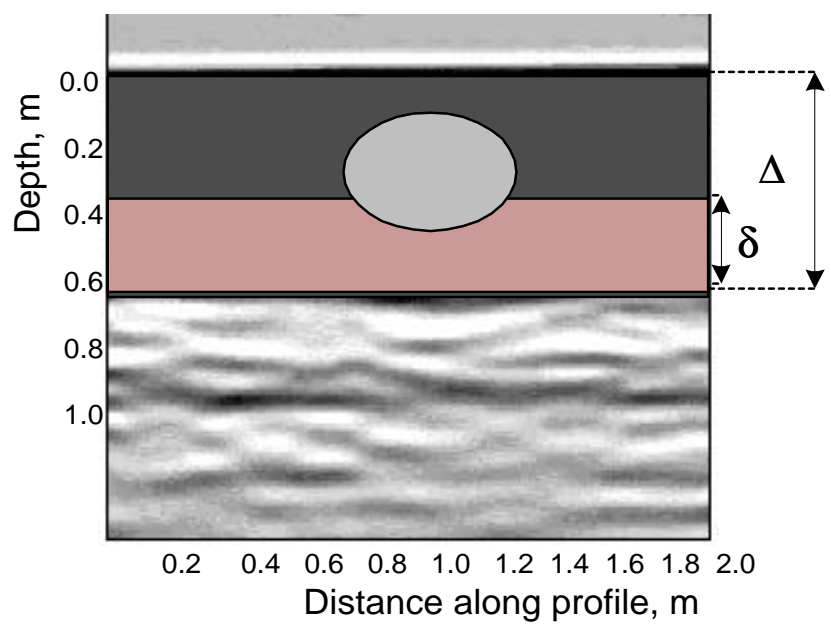

b)

Figure 3. Radar inspection to locate an old marble column hidden in brickwork: (a) initial radar image and (b) data interpretation. The Bandenelli Building, Lviv, Ukraine, 1999. 
The quantitative NDT approaches are aimed to estimate the geometrical properties of wall's internal regions. It is evident in Figures 2-4 that such kind of problem solution involve the wall thickness $\Delta$, the column diameter $\mathrm{D}$, the thickness of brickwork $\delta$ or plastered layers and others. Note that determination of the problem's spatial scaling like in Figures 2-4 must be based on the knowledge of exact magnitude of signal propagation velocity trough definite materials of walls. These values can be determined by the special calibration procedures based on reference data as well as direct measurements. Other structural elements inside the brick, limestone, concrete, wood walls have the potential to be characterized by radar like internal reinforcement discussed later.

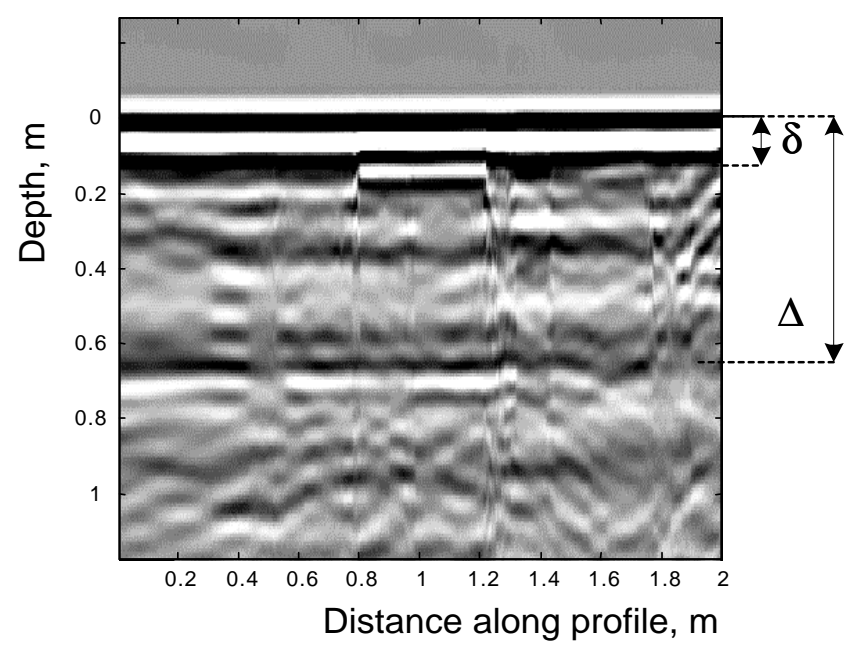

a)

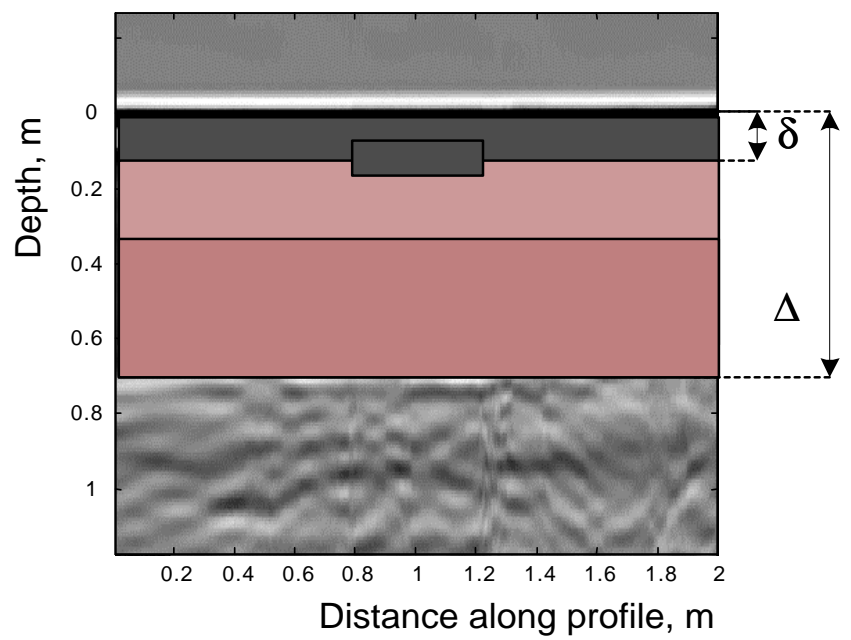

b)

Figure 4. Subsurface radar imaging of internal structure of the wall to locate the plastered niche. The Bandenelli Building, Lviv, Ukraine, 1999.

\section{SIGNAL PROCESSING TECHNIQUE}

As followed from previous discussion, a visual perception of electromagnetic images of wall's internal region by the expert is a key moment for both the qualitative and the quantitative NDT radar-based approaches. In this context psychological phenomena of human sighting and pattern recognition are more reliable and effective mean than computer ability to resolve similar tasks.

So image enhancement for its better visual perception should be a mandatory part of the radar processing software. Such required algorithms well known for optical image processing include statistical contrasting of radar returns to underline object borderlines, other form of statistical analysis, algorithms for detection of contour of hidden objects etc. The pseudo color model and modern computer graphic opportunities are also useful tools to process and visualize GPR data.

Some unavoidable obstacles and phenomena make worse the real radar images due to the inherent physical limitations and the achieved technical performances of available radar equipment. The last is mainly due to the used $1-4 \mathrm{GHz}$ frequency range. This operation band corresponds to the wavelength values of sounding signals that are comparable with the characteristic geometrical dimensions of the wall's internal structural elements.

Again transient-excited antennas in time-domain radar have wide-beam patterns. As result any radar return has serious diffraction features distorted the actual geometry of subsurface region under electromagnetic sounding. The image focusing techniques like F-K migration processing enables compensation of diffraction effects in radar.

At the same time a relatively long duration of directcoupling pulse in radar masks the near surface objects and features due to interference of such pulse and scattering signals generated by the near-surface plastered niche in Figure 4. The background direct-pulse removal processing is required to minimize the appointed undesirable effect.

Another complication of real radar image processing and interpretation is due to the weak contrast of electrical properties of internal structural materials like brick, mortar, concrete etc. For example, dielectric constant of such materials equals to $3 \ldots 6$ for low humidity levels $(\leq 5 \%)$.

A special set of the 1-D and 2-D signal processing procedures is necessary to compensate or minimize appointed undesirable effects and enhance the radar images of subsurface regions. As a mater of fact the optimal set of processing procedures should be chosen by heuristic reasons for definite problems due to complexity of its formal approval for very variable real operation conditions. 
Let illustrate signal processing for subsurface radar NDT by consideration a typical problem in building engineering to investigate the internal reinforcement of buildings such as walls, ceiling, floor etc. In this case the goal is to locate preciously internal position of steel/ferroconcrete reinforcement elements and estimate their geometry and corrosion degree if the last is possible.

Figure 6 demonstrates results of radar scanning on the floor of the thickness $\Delta$ in the building. Three clear visible diffraction events are caused by internal reinforcement in the floor. The next image in Figure $6 b$ is followed from previous one by its corresponding processing that includes the migration algorithm in a F-K spectrum domain. As result of such action the energy of all hyperbolic pattern is moved to the localized domains corresponded to actual position of the objects generated those diffraction patterns. Other useful moment is an opportunity to study detailed structure of reinforcement elements like shown in Figure 6c. By enhancing and enlarging the selected part of the processed before image the geometrical parameters of the reinforcement element as well as corrosion degree can be estimated. The last is determined by the characteristic of a halo around the bar.

It is also necessary to point out radar capabilities to detect objects located behind wall under investigation as seen in Fig. 6a. So, a set of invisible objects like heating tubes and others things under a floor in room below is fixed by radar located in another room above.

\section{CONCLUSIONS}

The presented study indicates on practical importance and necessity to use subsurface radar as effective tool for qualitative and quantitative NDT of the internal structure of walls and other building's brick, concrete, limestone, wood etc. elements. There are also some serious limitations forced by inherent and unavoidable physical factors like diffraction and large footprint of antennas. At the same time wide antenna beam is useful for searching and detection tasks. An optimal set of signal processing procedures is required to obtain high quality radar images of internal regions inside the building construction elements.

\section{REFERENCES}

Boryssenko, A.A., Boryssenko, O.S., 1999, "Determination of structure of columns, walls and fundaments by microwave sounding", Proc. of Conference LEOTEST'99, Kyiv, pp. 91-94. (In Russia).

Finkelstein, M.I. (Ed.), 1994, Subsurface Radars, Moscow, Radio i svjaz, 216 pp. (In Russia).

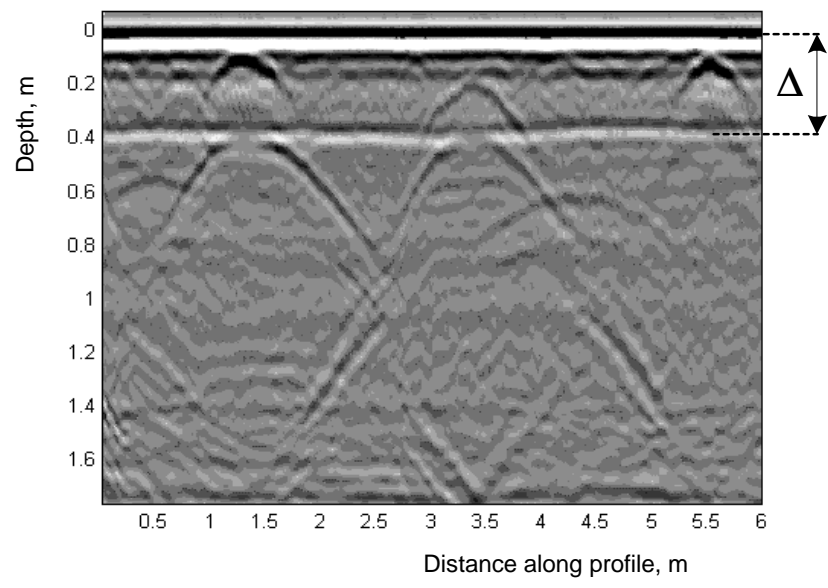

a)

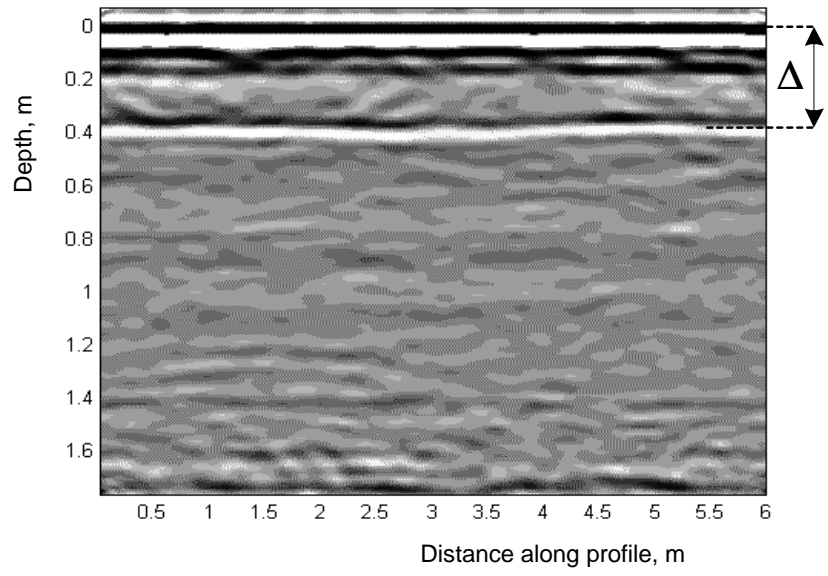

b)

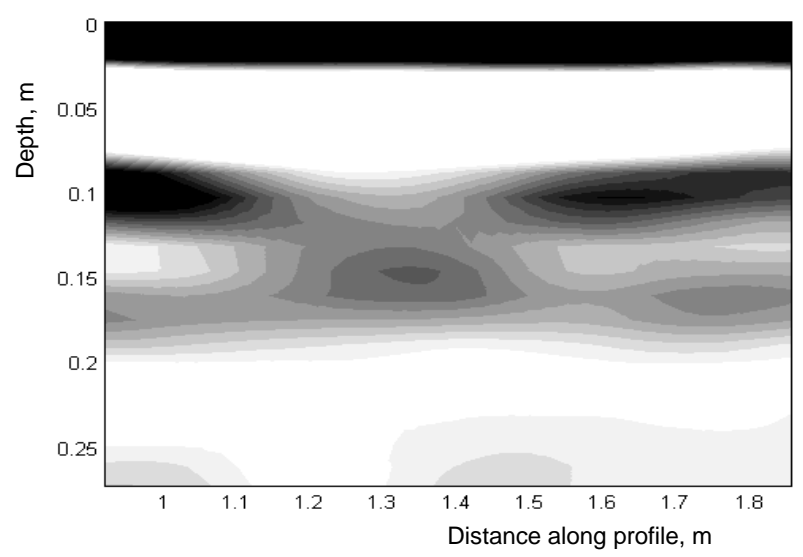

C)

Figure 5. Subsurface radar imaging of internal structure of wall to locate reinforcement bars: (a) initial image, (b) after processing, (c) enlarged fragment with reinforcement bar. The Odessa's State Opera Building, Odessa, Ukraine, 1997. 